\title{
INTEGRAL EXTENSIONS OF A RING
}

\author{
HAROLD CHATLAND AND H. B. MANN
}

Introduction. Let $R$ be a commutative ring with a unit element and let $a, b \subset R$.

Definitions. (1) $a$ and $b$ are said to be coprime in $R$ if $\tau \subset R$, $\tau / a, \tau / b$ implies $\tau / 1$.

(2) A ring $R^{\prime}$ is called an integral extension of $R$ if $R^{\prime} \supset R$ and $a=b r, a, b \subset R, r \subset R^{\prime}$ implies there exists an element $\bar{r} \subset R$ such that $a=b \bar{r}$.

(3) $a$ and $b$ are said to be absolutely coprime if they are coprime in every extension $R^{\prime}$ of $R$. In this paper it is shown that to every set of ideals of a commutative ring there exists an extension of the ring such that every ideal of the set is the intersection of the ring and a principal ideal of its extension. This is the main result and is given in Theorem 2. In a particular case of Theorem 2 it is shown in Theorem 1 that $a, b \subset R$ are absolutely coprime if and only if there exist elements $x, y \subset R$ such that $a x+b y=1$.

Similar results for algebraic integers are well known $[1] .{ }^{1}$ In the special case where the domains considered are completely integrally closed and the ideals have finite bases, a different extension fulfilling the conditions of Theorem 2 was obtained by Kronecker [2].

The extension of $R$. An extension of $R$, in the sense of this paper, may be obtained in the following manner. We first form the ring $R(u)$ by adjoining to $R$ the elements $u^{n}, n= \pm 1, \pm 2, \cdots$, transcendental over $R$ and such that $u^{n} a=a u^{n}, a \subset R$. Let $a$ be the ideal generated by the set $A$ of elements $a, b, \cdots$ of $R$. Then the subring $R^{\prime}$ of $R(u)$ consisting of all "polynomials"

$$
a_{-m} u^{-m}+a_{-m+1} u^{-m+1}+\cdots+a_{-1} u^{-1}+a_{0}+a_{1} u+\cdots+a_{n} u^{n}
$$

with $a_{i} \subset R$ and $a_{-r} \subset \mathfrak{a}^{r}, r>0$, is an integral extension of $R$ for $R^{\prime} \supset R$. Moreover if $c=d h, c, d \subset R, h \subset R^{\prime}$, then

$$
h=e_{-m} u^{-m}+\cdots+e_{-1} u^{-1}+e_{0}+e_{1} u+\cdots+e_{n} u^{n}
$$

$e_{i} \subset R, e_{-r} \subset \mathfrak{a}^{r}$. Multiplying by $d$ we have

$$
c=d e_{-m} u^{-m}+\cdots+d e_{-1} u^{-1}+d e_{0}+d e_{1} u+\cdots+d e_{n} u^{n} .
$$

Presented to the Society, November 28, 1947; received by the editors December 10, 1947, and, in revised form, April 19, 1948.

${ }^{1}$ Numbers in brackets refer to the references cited at the end of the paper. 
Since $c \subset R$, all terms involving $u$ must vanish. Hence $c=d e_{0}$ and $R^{\prime}$ is an integral extension of $R$.

\section{Results.}

THEOREM 1. $a, b \subset R$ are absolutely coprime if and only if there exist elements $x, y \subset R$ such that $a x+b y=1$.

Clearly the condition is sufficient. Use the above extension $R^{\prime}$ of $R$ where $A$ consists of $a$ and $b$. In $R^{\prime}=R\left(a u^{-1}, b u^{-1}, u\right), u$ is a common divisor of $a$ and $b$. If $a$ and $b$ are absolutely coprime, $u$ must be a unit divisor. Hence

$$
u^{-1}=\alpha_{-m} u^{-m}+\cdots+\alpha_{-1} u^{-1}+\alpha_{0}+\alpha_{1} u+\cdots+\alpha_{n} u^{n}
$$

$\alpha_{i} \subset R, \alpha_{-r} \subset \mathfrak{a}^{r}$. Since $u$ is trancendental over $R$ all terms in the sum must vanish except $\alpha_{-1} u^{-1}$. But $\alpha_{-1} \subset a$ and hence is of the form $a x+b y$. Multiplying by $u$ we have

$$
1=a x+b y \text {. }
$$

REMARK. If, $\bar{x}, \bar{y}$ are elements of $R$ such that

$$
a \bar{x}+b \bar{y}=1
$$

then the pair $x, y \subset R$ is also a solution if and only if $x=\bar{x}+b \mu$, $y=\bar{y}-a \nu$ where $a b \mu=a b \nu$.

Substitution shows the condition to be sufficient. Moreover if $a x+b y=1, \quad a \bar{x}+b \bar{y}=1$ then $a(x-\bar{x})=b(\bar{y}-y)$ and so $a \bar{x}(x-\bar{x})$ $=b(\bar{y}-y) \bar{x}$. Adding $b \bar{y}(x-\bar{x})$ to each side of the last equation we have

$$
x-\bar{x}=b(\bar{y}-y) \bar{x}+b \bar{y}(x-\bar{x})=b \mu .
$$

Similarly $y-\bar{y}=-a \nu$.

But $a(x-\bar{x})=b(\bar{y}-y)$. Hence

$$
a b \mu=a b \nu .
$$

LеммA. If $\mathfrak{a}$ is an ideal in $R$, then there exists an extension $R^{\prime}$ of $R$ such that $\mathfrak{a}$ is the intersection of $R$ and a principal ideal of $R^{\prime}$.

Consider the extension $R^{\prime}$ of $R$ with $\mathfrak{a}=A$. In $R^{\prime}$ the ideal $(u)$ is principal and contains $\mathfrak{a}$ and no other elements of $R$, for every element of $\mathfrak{a}$ is obtained from the set of products $a u^{-1} x u$. Also for $\lambda \subset R^{\prime}$ suppose $\lambda u=c, c \subset R$. Then $\lambda=c u^{-1}$. Hence $c \subset \mathfrak{a}$ and $\mathfrak{a}=R \wedge(u)$.

THEOREM 2. To every set of ideals of $R$ there exists an extension $R^{\prime}$ of $R$ such that every ideal of the set is the intersection of $R$ and a principal ideal of $R^{\prime}$. 
To each ideal $\mathfrak{a}$ in the set let there correspond an element $u_{\mathfrak{a}}$ transcendental over $R$. Form the ring $R(u)$ by adjoining $u_{\mathfrak{a}}^{n}$ to $R$ where $u_{\mathfrak{a}}^{n} a=a u_{\mathfrak{a}}^{n}, a \subset R, n= \pm 1, \pm 2, \cdots$. The subring $R^{\prime}$ of $R(u)$ consisting of the "polynomials"

$$
\sum a_{r_{1} r_{2} \ldots} u_{1}^{r_{1}} u_{2}^{r_{2}} \ldots, \quad \quad a_{r_{1} r_{2} \ldots \subset R},
$$

with the condition that $a_{r_{1} r_{2} \cdots r_{i}}$ belong to $\mathfrak{a}_{i}^{-r_{i}}$ if $r_{i}$ is negative, is an integral extension of $R$. This follows by the method demonstrated above. For if $l=m n, l, m \subset R, n \subset R^{\prime}$ then

$$
n=\sum \beta_{r_{1} r_{2}} \ldots u_{1}^{r_{1}} u_{2}^{r_{2}} \ldots
$$

where $\beta_{r_{1} r_{2}} \ldots$ are certain $a_{r_{1} r_{2}} \ldots$. Hence

$$
l=m n=\sum m \beta_{r_{1} r_{2}} \ldots u_{1}^{r_{1}} u_{2}^{r_{2}} \ldots .
$$

But since the indeterminates $u_{i}$ are transcendental over $R$ and $l \subset R$, all terms in the sum, except the constant term $m \beta_{0}$, must vanish. Hence $b=m \beta_{0}$.

We proceed as in the lemma. Consider the principal ideal (a). All of the elements $a^{(i)}$ of $\mathfrak{a}$ may be obtained as products $a^{(i)} u_{\mathfrak{a}}^{-1} \cdot u_{\mathfrak{a}}$. Moreover only the elements $a^{(i)} \subset R$ may be so obtained for suppose $\nu \subset R^{\prime}$ and $\nu u_{i}=d \subset R$. Then $\nu=d u_{i}^{-1}$. Hence $\mathfrak{a}=R \wedge\left(u_{i}\right)$.

REMARK. In the case of non-commutative rings a result analogous to Theorem 2 holds for two-sided ideals.

\section{REFERENCES}

1. H. Prufer, Neue Begrundung der algebraischen Zahlen Theorie, Math. Ann. vol. 94 (1925) pp. 198-244.

2. W. Krull, Idealtheorie, Ergebnisse der Mathematik und ihrer Grenzgebiete, 1935, pp. 124-127.

The Ohio State University 